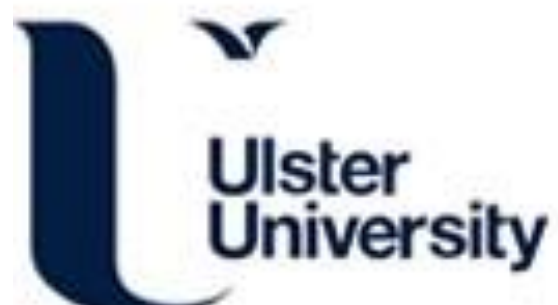

\section{Examining the Effect of General Practitioner Practice Size on Prescribing Behaviours in Northern Ireland}

Booth, F., Mulvenna, M., Bond, RR., McGlade, K., Rankin, D., \& Wallace, J. G. (2021). Examining the Effect of General Practitioner Practice Size on Prescribing Behaviours in Northern Ireland. In Proceedings - 2020 IEEE International Conference on Bioinformatics and Biomedicine, BIBM 2020 (pp. 2705-2708). (Proceedings - 2020 IEEE International Conference on Bioinformatics and Biomedicine, BIBM 2020). IEEE Xplore. https://doi.org/10.1109/BIBM49941.2020.9313570

Link to publication record in Ulster University Research Portal

Published in:

Proceedings - 2020 IEEE International Conference on Bioinformatics and Biomedicine, BIBM 2020

Publication Status:

Published (in print/issue): 13/01/2021

DOI:

https://doi.org/10.1109/BIBM49941.2020.9313570

\section{Document Version}

Author Accepted version

\section{General rights}

Copyright for the publications made accessible via Ulster University's Research Portal is retained by the author(s) and / or other copyright owners and it is a condition of accessing these publications that users recognise and abide by the legal requirements associated with these rights.

\section{Take down policy}

The Research Portal is Ulster University's institutional repository that provides access to Ulster's research outputs. Every effort has been made to ensure that content in the Research Portal does not infringe any person's rights, or applicable UK laws. If you discover content in the Research Portal that you believe breaches copyright or violates any law, please contact pure-support@ulster.ac.uk. 


\section{Examining the Effect of General Practitioner Practice Size on Prescribing Behaviours in Northern Ireland}

\author{
Frederick G Booth \\ School of Computing, \\ Ulster University Jordanstown \\ Northern Ireland, UK \\ booth-f@ulster.ac.uk \\ Kieran McGlade \\ Dunluce Family Practice \\ Belfast \\ kjmcglade@gmail.com
}

\author{
Maurice Mulvenna \\ School of Computing, \\ Ulster University Jordanstown \\ Northern Ireland, UK \\ md.mulvenna@ulster.ac.uk \\ Debbie Rankin \\ School of Computing, Engineering and \\ Intelligent Systems \\ Ulster University \\ Magee Campus \\ Northern Ireland, UK \\ d.rankin1@ulster.ac.uk
}

\author{
Raymond Bond \\ School of Computing, \\ Ulster University Jordanstown \\ Northern Ireland, UK \\ rb.bond@ulster.ac.uk \\ Jonathan Wallace \\ School of Computing, \\ Ulster University Jordanstown \\ Northern Ireland, UK \\ jg.wallace@ulster.ac.uk
}

\begin{abstract}
This study uses classifications of Metropolitan and Non-Metropolitan behavioural archetypes of General Practitioner practice in Northern Ireland and seeks to examine any associations between practice size and each archetype. It was found that the highest prescribing levels were in Small practices with two registered doctors for both archetypes. The lowest levels of prescribing were found in Single-Handed practices with only one registered doctor in Non-Metropolitan areas whilst Large practices with five or more registered doctors had the lowest prescribing levels in Metropolitan areas. One possible reason for Large practices having the lowest prescribing rates in Metropolitan areas may be the availability of more alternatives to medication. The highest prescribing levels in Non-Metropolitan areas were almost $4 \%$ higher than the lowest. This difference rose to $32 \%$ in Metropolitan areas. Further research into this difference is needed with deprivation levels found in Metropolitan areas being a possible factor. Examining each practice size individually showed that the same archetypes (i.e. Metropolitan and Non-Metropolitan) is also observed at this level with the number of registered patients increasing in line with the size of the practice although practices in Non-Metropolitan areas generally had more patients than those in Metropolitan areas.
\end{abstract}

Keywords-Health, General Practice, Open Data, Big Data, Prescriptions, Size

\section{Introduction}

Whilst the Institute for Fiscal Studies conducted research on the trends in General Practitioner practice size and the relationship between practice size and the quality of care experienced by patients [1], no studies have been identified examining the relationship between General Practitioner practice size and their associated prescribing levels. Building on previous work examining the link between geolocation and types of General Practitioner practice (the internal report detailing this process can be made available to researchers on request), this study will examine the relationship between different practice sizes and their prescribing levels. Previous work identified six features relating to the geolocation of GP practices and their relationship with associated pharmacies via the prescriptions issued / dispensed by them, and k-means clustering was performed which identified two distinct archetypes. These were classified as Metropolitan and NonMetropolitan, the former being in and around the city of Belfast, and the latter covering the rest of Northern Ireland. Details of the above process will be published in a different paper however this paper deals with the prescription levels associated with different sizes of GP practice, comparing levels found in both archetypes in order to gauge which is more efficient in terms of prescribing.

\section{Methods}

Using relatively simple statistical methods to provide insight into the prescribing behaviours of different sizes of GP practice, the number of registered GPs for each practice were obtained from the Northern Ireland Direct website [2] allowing each practice to be classified into one of four cohorts based on the size of the practice. These were: Single-Handed practices (1 doctor), Small practices (2 doctors), Medium sized practices (3-4 doctors and Large practices (5+ doctors). The number of items prescribed per registered patient was calculated for each practice over the period July 2015 to December 2019 and used to compare the different sizes of practice at both Northern Ireland level and within the two identified archetypes (Metropolitan and Non-Metropolitan). Each GP practice size was then examined using k-means clustering to discover what archetypes comprised therein. The same six features were used as in the previous study:

- Number of Pharmacies dispensing prescriptions issued by a practice.

- Average number of items prescribed per registered patient.

- Average distance travelled from a practice to dispense a prescription.

- Standard deviation of Distance travelled from a practice to dispense a prescription.

- Population density of the super output area in which the practice is located.

- Number of registered patients in the practice. 
Data on Practices, Pharmacies and the number of items dispensed was obtained from the Dispensing by Contractor datasets [3], distances were calculated using northings and eastings data relating to practice and pharmacy postcodes, population density was obtained from the Usual Resident Population dataset [4] and registered patients from GP Practice List Sizes [5]. Average number of items prescribed per registered patient was calculated in order to normalise this feature.

Finally, the average number of registered patients was calculated for each practice size in Northern Ireland and within each archetype in order to establish whether any differences existed.

\section{Results}

Comparing prescribing levels for the four sizes of GP practice at Northern Ireland level, it was found that there were no discernible differences in prescribing levels for SingleHanded, Medium and Large practices. Small practices however showed higher prescribing levels than the other three categories. (Figure 1)

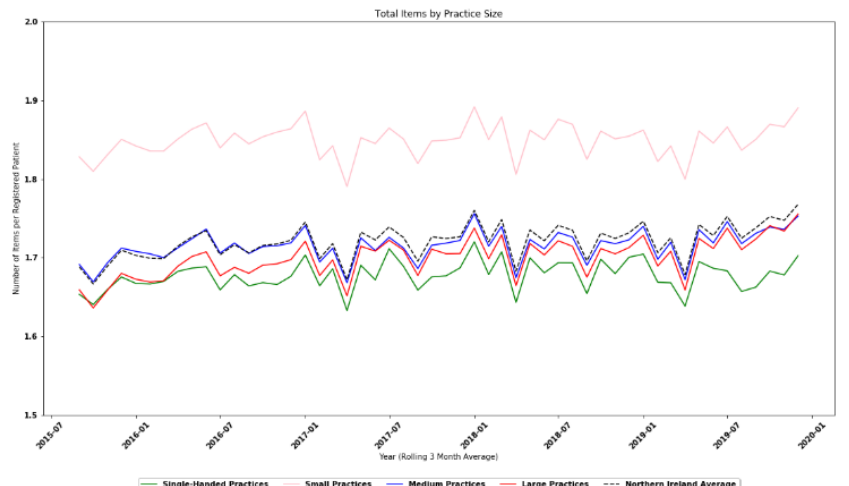

Figure 1: Prescribing levels in Northern Ireland by GP Practice size

Taking the average for each category it was found that Smalls had the highest prescribing levels (1.85 items per registered patient), 10\% higher than the lowest being Single-Handed practices (1.68 items per registered patient). Medium and Large practices prescribed 1.72 and 1.70 items respectively.

Comparing the number of practices in each category, it was found that of the 333 practices operating at March 2018 in Northern Ireland, $29(8.7 \%)$ were Single-Handed practices, $61(18.3 \%)$ Small practices, 122 (36.6\%) Medium practices and $121(36.3 \%)$ Large practices. Splitting these practices into their behavioural archetypes, there were 90 Metropolitan and 243 Non-Metropolitan practices. Both archetypes were found to be of similar proportions to that seen for Northern Ireland (Figure 2).

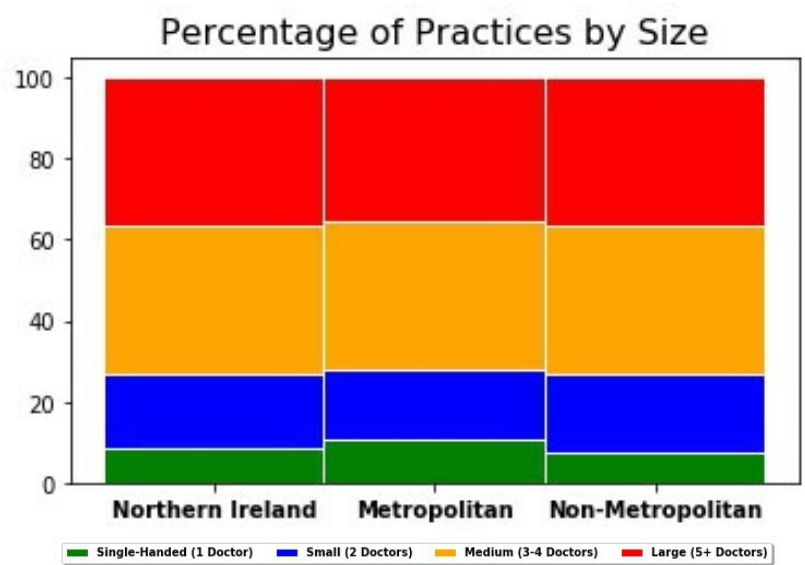

Figure 2: Percentage of Practices by Size

Examining each archetype in turn, it was found that within the Metropolitan area prescribing levels were highest in Small practices ( 2.21 items per registered patient), 32\% higher than in Large practices (1.67 items per registered patient). SingleHanded and Medium practices prescribed on average 1.76 and 1.81 items respectively (Figure 3 )

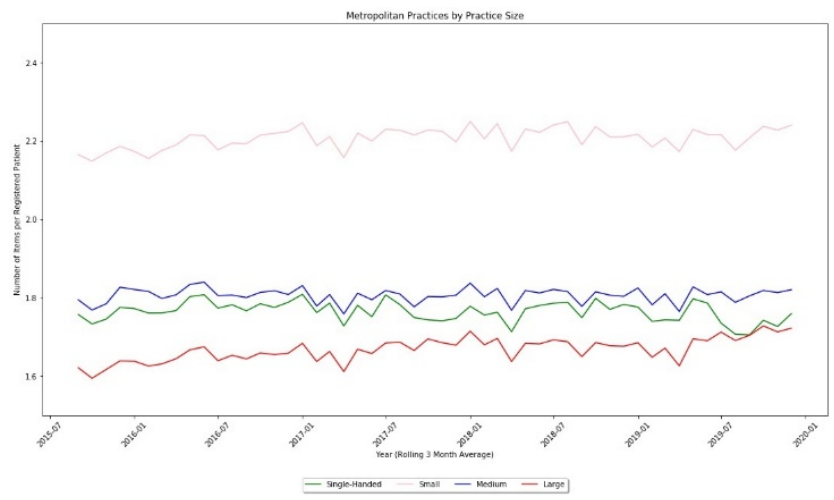

Figure 3: Prescribing levels for Metropolitan practices by size

Within the Non-Metropolitan area prescribing levels again were highest in Small practices (1.74 items per registered patient), 3.8\% higher than in Single-Handed practices (1.64 items per registered patient). Medium and Large practices prescribed on average 1.68 and 1.71 items respectively (Figure 3).

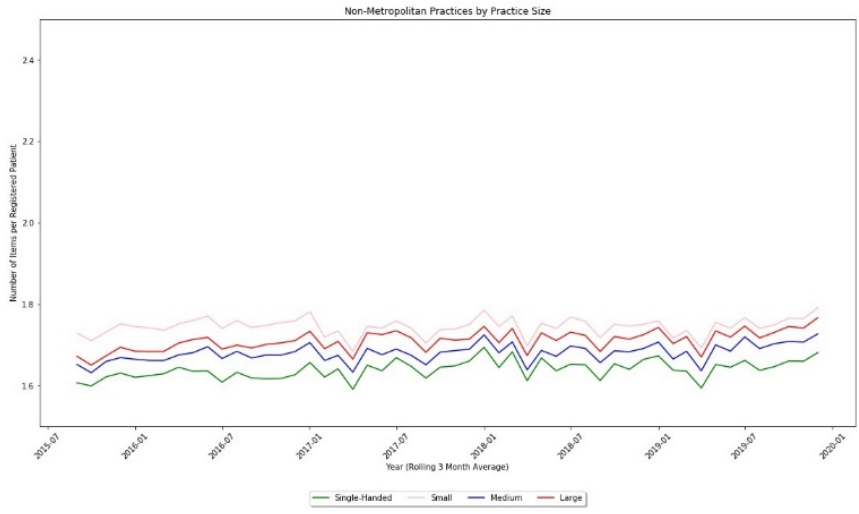

Figure 4: Prescribing levels for Non-Metropolitan practices by size 
Examining GP practices within the four size bands using kmeans clustering, it was found that regardless of GP practice size the clustering identified across all GP practice types also held for each practice size. It was found that two of the features (Number of Pharmacies and Distance Travelled) contributed most to the variations between archetypes.

Single-Handed practices in the Non-Metropolitan area typically had 67 pharmacies dispensing their prescription with an average distance of 13 kilometres being travelled to a pharmacy. Practices in the Metropolitan area had 161 pharmacies with an average distance of 4 kilometres being travelled (Figure 5).
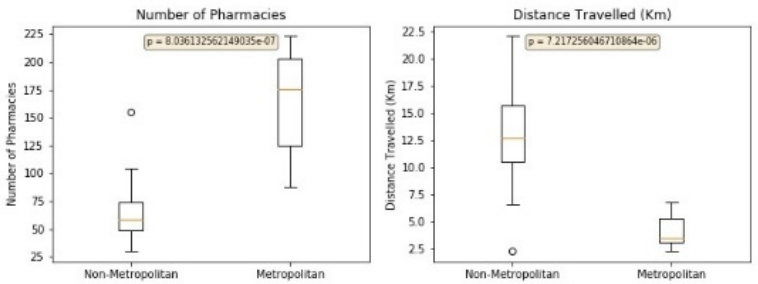

Figure 5: Number of Pharmacies and Distance Travelled for Single-Handed practices.

Small practices in the Non-Metropolitan area typically had 75 pharmacies dispensing their prescription with an average distance of 15 kilometres being travelled to a pharmacy. Practices in the Metropolitan area had 199 pharmacies with an average distance of 4 kilometres being travelled (Figure 6).
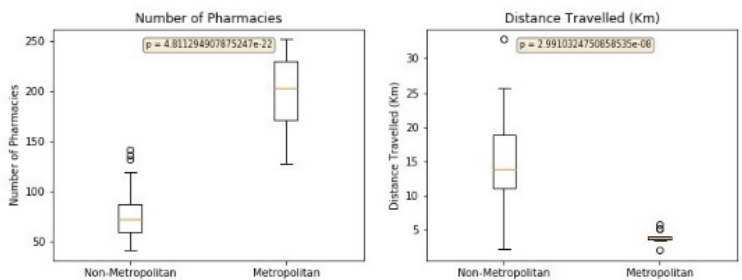

Figure 6: Number of Pharmacies and Distance Travelled for Small practices.

Medium sized practices in the Non-Metropolitan area typically had 94 pharmacies dispensing their prescription with an average distance of 15 kilometres being travelled to a pharmacy. Practices in the Metropolitan area had 211 pharmacies with an average distance of 4 kilometres being travelled (Figure 7).
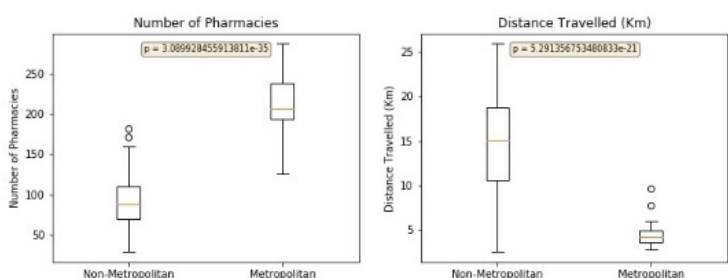

Figure 7: Number of Pharmacies and Distance Travelled for Medium sized practices.

Large practices in the Non-Metropolitan area typically had 121 pharmacies dispensing their prescription with an average distance of 15 kilometres being travelled to a pharmacy. Practices in the Metropolitan area had 221 pharmacies with an average distance of 6 kilometres being travelled (Figure 8).
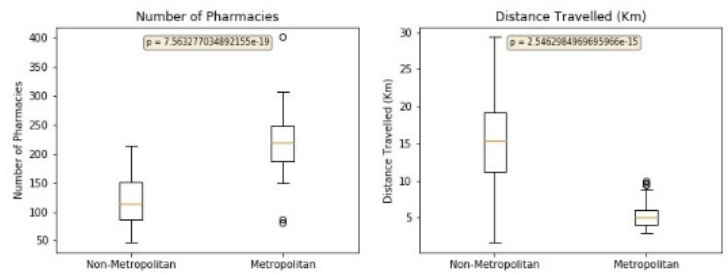

Figure 8: Number of Pharmacies and Distance Travelled for Large practices.

Examining the number of registered patients for each practice type, the Northern Ireland average is 2,983 for Single-Handed practices, 2,677 for Small practices, 5,098 for Medium practices and 8,600 for Large practices. With the exception of Medium sized practices, practices in Non-Metropolitan areas have more registered patients than their respective counterparts in Metropolitan areas. (Figure 9)

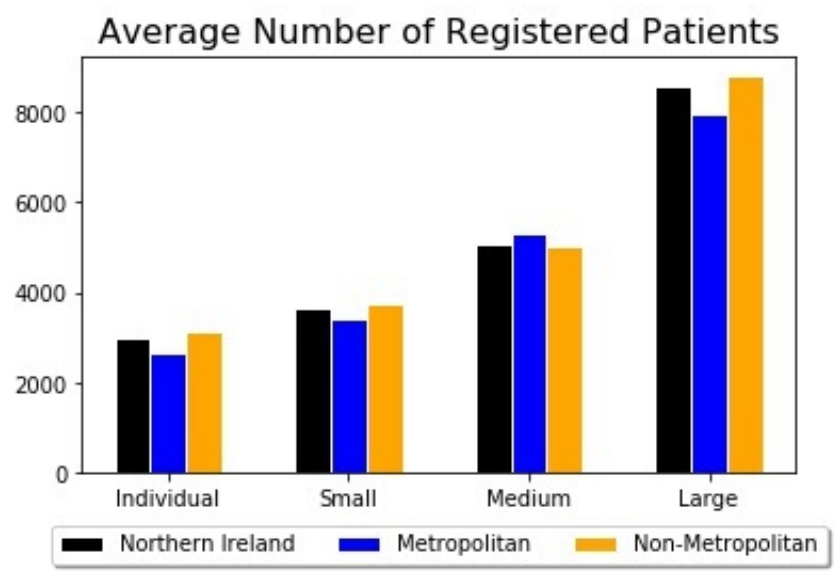

Figure 9: Average Number of Registered Patients by GP Practice Size

\section{Discussion}

Comparing prescription levels of different sized General Practitioner practices at Northern Ireland level shows that the highest prescribing cohort are Small practices with two registered doctors ( 1.85 items per registered patient). This cohort prescribes on average 10\% more than Single-Handed practices with only one registered doctor being the lowest prescribing cohort (1.68 items per registered patient). Medium and Large practices (3-4 and 5+ doctors respectively) had similar prescribing levels of 1.72 and 1.70 items per registered patient respectively. These results disprove the general theory that Single-Handed practices are more likely to have higher prescription rates due to the pressures on an individual doctor running a practice.

Comparing the two archetypes (Metropolitan and NonMetropolitan) it is evident that Small practices are the highest prescribing cohorts in both archetypes with Large practices having the lowest prescribing levels in Metropolitan areas and Single-Handed practices in NonMetropolitan areas. It is interesting to note that the difference in prescribing levels is not as pronounced in practices in Non-Metropolitan areas with highest prescribing being 3.8\% higher than the lowest. In contrast, the highest prescribing 
levels for practices in Metropolitan areas is 32\% higher than the lowest. Analysis of each of the four sizes of practice showed that these conformed to the two archetypes previously established reinforcing those findings. Further research is needed to explain why a larger difference is seen in practices in Metropolitan areas although deprivation is a possible factor. The lowest prescribing levels in practices in Non-Metropolitan areas are seen in Single-Handed practices. This may be due to the relatively larger number of registered patients in these practices along with a greater knowledge of their patients which will influence prescribing. Prescribing in Small and Medium practices allows the possibility of less tight control on prescribing and they also lack the advantages of scale that Large practices have. The lowest prescribing levels in Metropolitan areas are seen in Large practices which have the advantage of scale and availability of extra services e.g. Cognitive Behaviour Therapy.

\section{Limitations}

Practices have been categorised based on the number of registered doctors working in the practice. The assumption has been made that all of these doctors work full-time which in reality is probably not the case. Also, as no data is available on the number of locums working in any practice, these have been ignored.

\section{Conclusion}

Comparing prescribing levels of different sizes of GP practice at Northern Ireland level and within the two previously identified archetypes of GP practice shows that higher prescribing levels are consistently associated with Small practices (two registered doctors). Whilst these practices' prescribing levels are, on average, $10 \%$ higher than the lowest prescribing practices in Northern Ireland, levels in Metropolitan areas are 32\% higher with NonMetropolitan areas being 3.8\%. Further research into the higher levels seen in Metropolitan areas with a possible factor being the level of deprivation attributed to these areas. Overall, the lowest prescribing levels in Northern Ireland are seen in Single-Handed practices with only one registered doctor and may be attributed to a better knowledge of patients influencing prescribing. This is also seen in practices in Non-Metropolitan areas but interestingly, the lowest prescribing practices in Metropolitan areas are Large practices with five or more registered doctors which benefit from the economies of scale and the possible provision of extra services being available.

\section{REFERENCES}

[1] Kelly, E. (n.d.). Does GP Practice Size Matter? GP Practice Size and the Quality of Primary Care.

[2] HSC Business Services Organisation (2020) GP Practice List Sizes, Available at: https://www.opendatani.gov.uk/dataset/gp-practice-listsizes (Accessed: 28 April 2020).

[3] HSC Business Services Organisation (2020) Dispensing by Contractor, Available at: https://www.opendatani.gov.uk/dataset/dispensing-bycontractor (Accessed: 28 April 2020).
[4] Office for National Statistics (2014) Usual resident population, Available at: https:/www.nomisweb.co.uk/census/2011/ks101uk (Accessed: 28 April 2020).

[5] HSC Business Services Organisation (2020) GP Practice List Sizes, Available at: https://www.opendatani.gov.uk/dataset/gp-practice-listsizes (Accessed: 28 April 2020). 